\title{
Contenido de As, Ba, y Cu en sedimentos y su asociación con depósitos minerales en el noreste de Chihuahua
}

\author{
As, $\mathrm{Ba}$, and $\mathrm{Cu}$ content in sediments and their \\ association with mineral deposits of northeast \\ Chihuahua
}

MÉlida Gutiérrez, ${ }^{1,3}$ Enrique Carreon, ${ }^{2}$

Recibido: Abril 15, 2008

Aceptado: Agosto 14, 2008

\section{Resumen}

La distribución de arsénico (As), bario $(\mathrm{Ba})$, y cobre $(\mathrm{Cu})$ en sedimentos se analizó con el fin de determinar su posible asociación con depósitos minerales en el noreste de Chihuahua. El estudio se basó en 1,016 muestras de sedimento de arroyo y 11 de sedimento de río. Concentraciones enriquecidas y anómalas de cada uno de estos tres elementos se compararon con la ubicación geográfica de depósitos minerales de sulfatos, sulfuros ( $\mathrm{Pb}-\mathrm{Zn}-\mathrm{Cu}$ ) y óxidos de Cu y Mn utilizando el sistema de información geografica ArcGIS. La concentración natural de fondo para cada elemento correspondió a concentraciones menores al percentil 80 y los valores anómalos a concentraciones mayores que el promedio más dos desviaciones estándar. A las concentraciones intermedias (valores no-anómalos y > percentil 80) se les denominó concentraciones naturales enriquecidas. Las distribuciones de concentraciones naturales, naturales enriquecidas, y anómalas presentaron un patrón de distribución diferente para cada elemento. Un patrón de comportamiento común a los tres elementos fue la relación entre concentraciones anómalas y la presencia de depósitos minerales.

Los valores de fondo naturales y enriquecidos se pueden utilizar como base en el monitoreo de cambios en la composición química del área resultantes de actividades mineras y agrícolas, mientras que la identificación de concentraciones anómalas se debe considerar como lugares en donde contaminación está ocurriendo a mayor escala y donde se requiere de algún tratamiento de contención o remediación de contaminantes.

Palabras clave: Arsénico, bario, cobre, concentración anómala, contaminación, Chihuahua, sedimento.

\begin{abstract}
Concentrations of arsenic (As), barium (Ba) and copper (Cu) in arroyo sediments were statistically and spatially analyzed to determine their relationship with mineral deposits occurring in northeast Chihuahua.

The study utilized 1,016 arroyo sediment samples and 11 river sediment samples. Their spatial distribution, obtained using ArcGIS, was compared to the geographical location of known sulfates, sulfide $(\mathrm{Pb}-\mathrm{Zn}-\mathrm{Cu})$ and $\mathrm{Mn}$-oxide mineral deposits

The natural background concentration values corresponded to values $<80 \%$ percentile while anomalous values were identified as those larger than the mean plus two times the standard deviation. The rest of the values (non-anomalous values $>80 \%$ percentile) were labeled as naturally enriched. The distributions of natural, enriched and anomalous concentrations yielded a different pattern for each element. A pattern common to all elements was that anomalous concentrations clustered mineral deposits. Natural background concentrations and background enriched concentration can be used as reference values to monitor chemical changes that may occur due to agriculture or mining activities, while the identification of anomalous concentrations should be considered as a location where pollution is taking place and where a containment or remediation treatment is required.
\end{abstract}

Key words: Arsenic, barium, copper, anomalous concentration, pollution, Chihuahua, sediment.

\footnotetext{
${ }_{1}^{1}$ Departamento de Geografía, Geología y Planeación, Universidad del Estado de Missouri, Springfield MO 65897 USA

${ }^{2}$ PROFAUNA, Calle 16 No. 2604 Col. Pacífico, Chihuahua, Chih. 31030, México

${ }^{3}$ Dirección electrónica del autor de correspondencia: mgutierrez@missouristate.edu
} 


\section{Introducción}

I uso de sedimentos como indicadores de contaminación en estudios ambientales, incluyendo el índice de geoacumulación propuesto por Mueller (1979) y el fraccionamiento por fases químicas para determinar la movilidad de contaminantes propuesto por Tessier et al. (1979) siguen siendo utilizados por un gran número de investigadores, especialmente por la propiedad de los sedimentos de retener información de los contaminantes durante un lapso de tiempo mayor comparado con las fases acuosas (Förstner y Wittmann, 1981).

Una posible desventaja en la utilización de sedimentos es el aspecto analítico, ya que el análisis es más elaborado que el de soluciones acuosas debido a que la muestra se tiene que someter a tratamientos de secado, tamizado y digestión antes de su análisis por medio de espectrometría, esta última utilizando espectrómetros de absorción atómica o de inducción de plasma. Los resultados obtenidos se expresan comúnmente en $\mathrm{mg}$ de contaminante por $\mathrm{kg}$ de sedimento seco, o ppm.

Bases de datos geoquímicos de sedimentos han proliferado en las últimas décadas como resultado de programas nacionales y multinacionales, como el Mapeo Geoquímico Mundial (GRN por sus siglas en ingles de Global Reference Network) y el Proyecto Geoquímica de Suelos y Paisajes de Norteamérica (Geochemical Landscapes Project), el cual

Figura 1. Área de estudio mostrando la ubicación de minas existentes, seis de las cuales se encuentran en operación y están marcadas con un rombo; las demás minas están abandonadas. Picos rojos se utilizaron para distinguir minas de metales asociados con Cu y As, negros para barita, y grises para otros minerales. La línea gruesa gris demarca la cuenca del Rio Conchos.

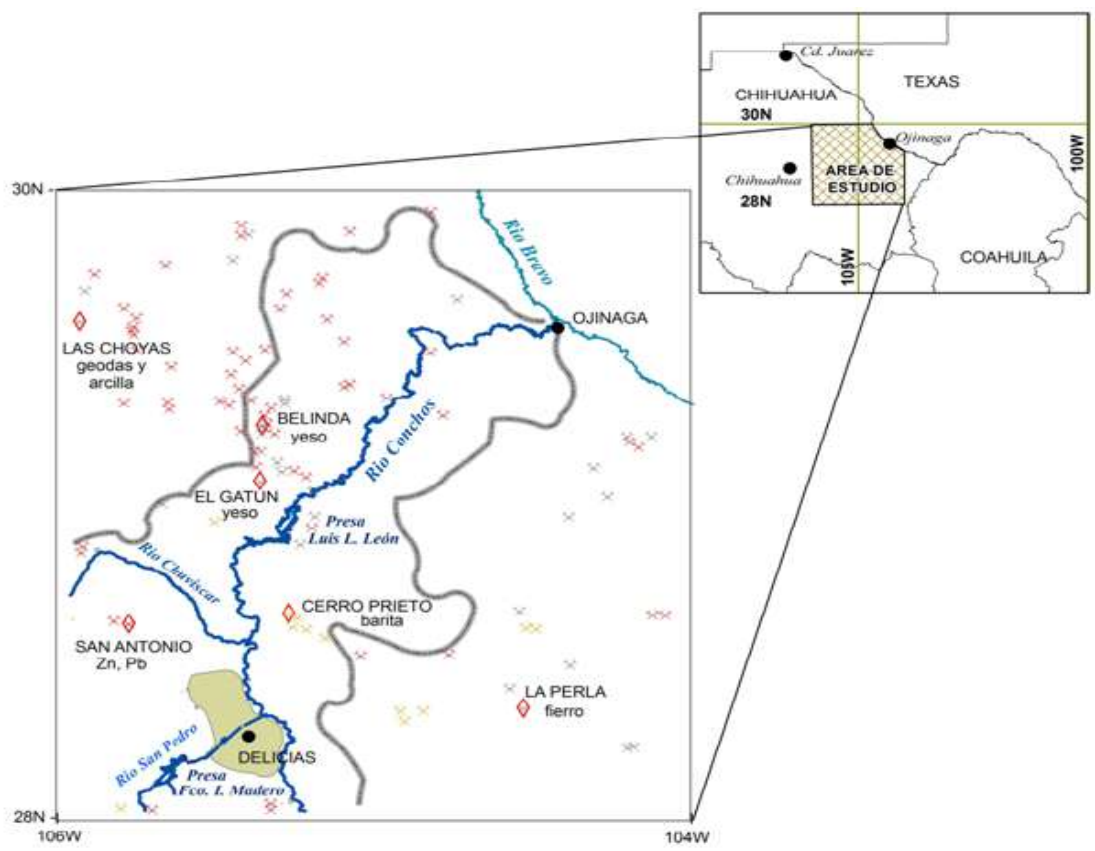


Mélida Gutiérrez, Enrique Carreon: Contenido de As, Ba, y Cu en sedimentos y su asociación con depósitos minerales en el noreste de Chihuahua

tiene como objetivo la cobertura de Estados Unidos, Canadá y México. El Servicio Geológico Mexicano ha acumulado un banco de datos, los cuales están disponibles en cuadros de $1^{\circ} \times 2^{\circ}$. Para los cuadros de Delicias y Ojinaga en el estado de Chihuahua, muestras de sedimentos se colectaron en 1999 y los resultados se publicaron en 2001 (SGM, 2001). Estos dos cuadros, los cuales cubren el área entre $24-26^{\circ} \mathrm{N}$ y $104-106^{\circ} \mathrm{W}$ (Fig. 1), contienen un total de 1,016 puntos de muestreo bajo una cobertura bastante buena, considerando que partes del área son de difícil acceso.

Este estudio se basó en 1,027 concentraciones de $\mathrm{As}, \mathrm{Ba}, \mathrm{y} \mathrm{Cu}$ en sedimentos; 1,016 muestreados y analizadas por SGM y 11 muestras muestreadas por los autores y analizadas en un laboratorio comercial (CHEMEX) utilizando protocolos estándar de análisis de metales descritos más adelante.

As, $\mathrm{Ba}, \mathrm{y} \mathrm{Cu}$ fueron seleccionados para este estudio por ser elementos potencialmente tóxicos, por su presencia en depósitos minerales del área, y por tener un número considerable de muestras arriba de límites de detección para así lograr un análisis estadístico más representativo.

Bario

El bario afecta la salud humana ya sea por ingestión o inhalación; en dosis alta afecta el sistema nervioso y los riñones (ATSDR, 2007), y en concentraciones extremas (contacto prolongado con suelos $>1,400 \mathrm{ppm}$ $\mathrm{Ba})$ se ha llegado a relacionar con parálisis y esclerosis múltiple (Purdey, 2004). El sulfato y el carbonato de bario son sales poco solubles y de baja toxicidad. Los depósitos de Ba se encuentran solos o asociados con depósitos de $\mathrm{Zn}, \mathrm{Pb}$, u óxidos de manganeso (Mosier 1986; Paradis et al., 1998).

El promedio mundial reportado para suelos es de 430 ppm Ba (Breckenridge y
Crockett, 1995). La norma oficial para $\mathrm{Ba}$ en suelos de uso agrícola y comercial es de 5,400 ppm (SEMARNAT, 2007). Para agua potable, la norma oficial mexicana es 0.7 ppm (DOF, 1996). Según un estudio llevado a cabo por Wones et al., (1990) un grupo de personas ingirió agua conteniendo 10 ppm de Ba durante 10 semanas sin presentarse ningún efecto adverso.

\section{Cobre}

El cobre es un metal esencial para organismos vivos en cantidades pequeñas, pero tóxico en concentraciones altas (Flemming y Trevors, 1989). En aguas naturales, $\mathrm{Cu}$ afecta a los organismos acuáticos en mayor o menor grado dependiendo del organismo, química del agua, pH, y tiempo de exposición. En agua, valores alrededor de $42 \mathrm{ppm} \mathrm{Cu}$ afectaron a Ceriodaphnia dubia, un organismo utilizado como indicador de calidad, (EPA, 2001). En cuanto a sedimentos, Ios organismos acuáticos empiezan a mostrar afectación (nivel bajo) a partir de 70 ppm Cu, mientras que un contenido de 390 ppm Cu, considerado nivel medio, causó mortandad a aproximadamente la mitad de ellos (Long y Morgan, 1990). El valor promedio de Cu en suelos se ha reportado como 30 ppm (Breckenridge y Crockett, 1995). La norma oficial mexicana para agua potable es de 2.0 ppm (DOF, 1996).

\section{Arsénico}

En contraste con el bario y el cobre, el arsénico es tóxico a concentraciones muy bajas y además es insípido e incoloro; la norma oficial mexicana para agua potable es de 0.025 ppm (SEMARNAT, 1996) y para suelos de uso agrícola es 22 ppm (DOF, 2007). En sedimentos, el valor bajo para afectación de organismos acuáticos es de 33 ppm y 85 ppm para nivel medio (Long and Morgan, 1990). 
Mélida Gutiérrez, Enrique Carreon: Contenido de As, Ba, y Cu en sedimentos y su asociación con depósitos minerales en el noreste de Chihuahua

\section{Antecedentes}

Los arroyos dentro del área de estudio representan en su mayoría condiciones naturales ya que prácticamente todas las substancias de desecho de ciudades, drenes agrícolas, etc., son descargados a los ríos Conchos, Chuvíscar, y San Pedro. La afinidad de metales y metaloides por adsorberse en fases sólidas sugiere una inevitable acumulación en sedimentos en estos ríos y sus reservorios. Comparado con los ríos, la reducción en la velocidad del agua en reservorios causa un asentamiento de partículas finas (arcillas) en los sedimentos, y debido a que éstas últimas están enriquecidas con As y $\mathrm{Cu}$, la concentración de As y Cu en los sedimentos se incrementa (Hernández-García et al., 2007; Gutiérrez et al. , en prensa; Gutiérrez et al., 2008). El arsénico en sedimentos y ríos en Chihuahua se ha asociado a jales de minas y descargas de aguas residuales (Gutiérrez et al., 2008; Gutiérrez-Ruiz et al., 2007).

En las inmediaciones de jales, las concentraciones de metales y otros productos de minas pueden ser muy altos (Puga et al., 2006; Gutiérrez-Ruiz et al., 2007). Gutiérrez-Ruiz et al. (2007) reportaron valores de $\mathrm{Cu}, \mathrm{As}$, y Ba de hasta 2,415 mg/kg, 3,281 mg/kg y $586 \mathrm{mg} / \mathrm{kg}$ respectivamente en la zona minera de Santa Barbara (Chihuahua) y sin embargo su contenido en aguas subterráneas someras se encontró por debajo de los límites señalados para aguas de consumo humano.

Debido a la afinidad de metales y metaloides (As, Cu) para ser retenidos por fases sólidas, su concentración más alta se espera encontrar cerca de minas y jales, decreciendo con la distancia (Puga et al., 2006), mientras que elementos que son no son tan fácilmente adsorbidos por la fase sólida (e.g., Ba) tienden a dispersarse y ser transportados con las escorrentías.

Área de estudio

El área de estudio se encuentra entre $28-30^{\circ} \mathrm{N}$ y $104-106^{\circ} \mathrm{W}$ (Fig. 1). Delicias $(127,211$ habitantes) y Ojinaga $(21,157$ habitantes) son ciudades importantes dentro del área; otras poblaciones incluyen Julimes, Coyame, Aldama, Cárdenas, y Cuchillo Parado. El área está comprendida dentro del desierto de Chihuahua y tiene una elevación promedio de 1,100 msnm. Dentro del área de estudio hay dos distritos de riego, el Distrito 005 (Delicias) con una superficie de 84,528 hectáreas y el Distrito 090 (Ojinaga) con 10,975 hectáreas (INEGI, 1999).

La precipitación es de tipo monzón, siendo julio, agosto y septiembre los meses de lluvia. La precipitación anual es variable, desde 140 mm (2001) hasta 601 mm (1978). Dentro del área existen gran cantidad de arroyos, los cuales permanecen secos gran parte del tiempo. Los ríos principales son el Conchos, Bravo, Chuvíscar y San Pedro. Los dos últimos desembocan en el río Conchos, y el río Conchos desemboca en el río Bravo. Dentro del área existen 98 minas, de las cuales solamente seis están operando: Belinda y El Gatun (yeso), SanAntonio (metales: plomo y zinc), Cerro Prieto (barita), La Perla (fierro) y Las Choyas (arcillas y geodas) (SGM, 2001). La relación entre estos depósitos minerales y su geología se ha reportado por diversos autores (Clark y De la Fuente, 1978; Arzabala-Molina, 2005). La ubicación de estas minas se muestra en Fig. 1. Para facilitar su identificación visual, los depósitos de metales asociados con $\mathrm{Cu}$ y As (e.g., Zn, Pb) están representadas por picos color rojo, y minerales conteniendo $\mathrm{Ba}$ por picos color negro.

El acceso al área de estudio por tierra es restringido ya que solamente dos 
carreteras la cruzan, las No. 45 (Panamericana) y No. 16 (ChihuahuaOjinaga). El resto del área está comunicado por medio de caminos de terracería, la mayoría de los cuales se vuelven intransitables durante temporadas de lluvia.

Métodos

Muestras de sedimento se tomaron de la parte superficial del lecho de arroyos durante temporada de estiaje, se secaron a temperatura ambiente, y se pasaron por una criba 80 mesh. Muestras de 1 gramo del sedimento seco tamizado se digirieron con agua regia. La solución resultante se analizó en un espectrómetro de inducción de plasma (ICP-MS) en los laboratorios del SGM en Pachuca, Hidalgo, siguiendo protocolos estándar (SGM, 2001). Las muestras colectadas por los autores se sometieron al mismo protocolo seguido por el SGM, excepto que las muestras se tomaron del lecho del río cubierto por agua, aproximadamente a $1 \mathrm{~m}$ de la orilla, y se enviaron a un laboratorio comercial (CHEMEX; http://www.alsglobal.com/) para su análisis.

Una vez que se compilaron las concentraciones, el primer paso en el análisis de concentraciones fue construir un histograma (frecuencia vs concentración) para cada elemento. Los histogramas mostraron una distribución típica de componentes de concentración natural y enriquecido (Voigt et al., 2005), la cual consiste en una distribución normal para concentraciones bajas seguidas por una segunda curva de campana para las concentraciones enriquecidas. El histograma obtenido para Cu se muestra en la Fig. 2.

Figura 2. Histograma de concentraciones de cobre mostrando dos componentes; concentraciones naturales de fondo (línea naranja) y concentraciones enriquecidas (línea verde).

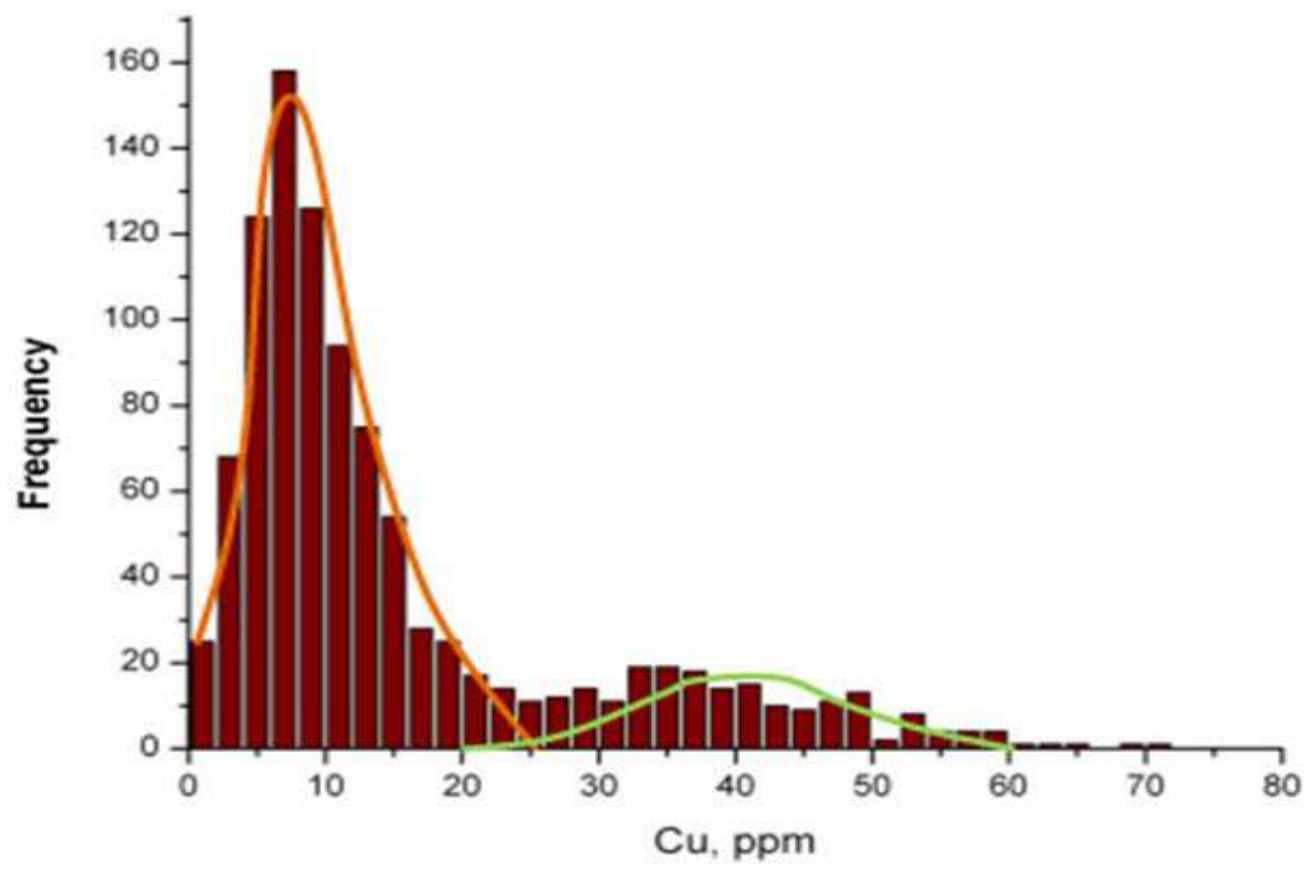


Las concentraciones naturales de fondo se obtienen generalmente utilizando el percentil 90 de las muestras (San Juan, 1994) y tienen una distribución normal. Dado que la distribución obtenida para nuestras muestras presentó por lo menos dos componentes, se determinó por inspección visual la concentración máxima de acuerdo a una distribución normal (primera curva de campana), con un valor aproximado de 25 ppm (ver Figura. 2).

Esta concentración se comparó con los valores de concentraciones listados de menor a mayor, encontrándose que $20 \%$ de las muestras correspondieron a concentraciones $>25 \mathrm{ppm}$, por lo cual se seleccionó el percentil 80 para separar las muestras enriquecidas ( $>$ percentil 80 ) de las concentraciones de fondo natural (< percentil 80). Similarmente, concentraciones anómalas se obtuvieron al separar aquellos valores mayores que el promedio más dos desviaciones estándar (Cuadro 1). En base a estos valores se construyeron mapas de la distribución de cada tipo de concentración (natural, natural enriquecida, y anómala) para cada uno de los tres elementos.

Cuadro 1. Valores promedio, desviación estándar, y numero de muestras correspondientes a concentraciones de fondo natural (< percentil 80 ), enriquecidas ( ${ }^{3}$ percentil 80 ) y anómalas.

\begin{tabular}{cccc}
\hline & $\mathrm{Ba}$ & $\mathrm{Cu}$ & $\mathrm{As}$ \\
\hline Percentil 80, ppm & 253.8 & 24.9 & 9.6 \\
Concentración de & $160.2 \pm 51.1$ & $9.4 \pm 5.0$ & $6.1 \pm 1.7$ \\
fondo natural, ppm & $\mathrm{N}=822$ & $\mathrm{~N}=823$ & $\mathrm{~N}=820$ \\
Concentración & $344.8 \pm 103.6$ & $41.2 \pm 17.1$ & $17.6 \pm 18.5$ \\
enriquecida, ppm & $\mathrm{N}=205$ & $\mathrm{~N}=204$ & $\mathrm{~N}=207$ \\
Valores anómalos, & $>363.8$ & $>46.6$ & $>27.5$ \\
Ppm No. valores & 42 & 51 & 18 \\
anómalos & & & \\
\hline
\end{tabular}

\section{Discusión de resultados}

Los mapas para concentraciones naturales y naturales enriquecidas se muestran en las Figuras. 3 - 5. A simple vista, los mapas de valores naturales de fondo (< percentil 80) mostraron la distribución de $\mathrm{Cu}$ ligeramente concentrada en la parte norte del área de estudio. Al verificar la diferencia en la distribución de cada uno de los elementos en zonas norte y sur por medios estadísticos (G-test de variosimilitud) se comprobó que $\mathrm{Cu}$ y $\mathrm{Ba}$ están más densamente distribuidos en una de estas zonas, el $\mathrm{Cu}$ en el norte y el $\mathrm{Ba}$ en el sur (Cuadro 2).

Los valores anómalos se consideran en general como el resultado de factores operando fuera de los procesos naturales, como es el caso de la contaminación de tipo antropogénica. Los valores límite para concentraciones anómalas, así como el número de concentraciones anómalas obtenidos para cada elemento se incluyen en el Cuadro 1. 
Figura 3. Concentraciones de fondo (mapa superior) y enriquecidas (mapa inferior) para As. Las minas están marcadas con cruces (rojas para óxidos, moradas para sulfuros). El mapa muestra además el río Conchos y su cuenca, y el sistema de riego 005 (verde).

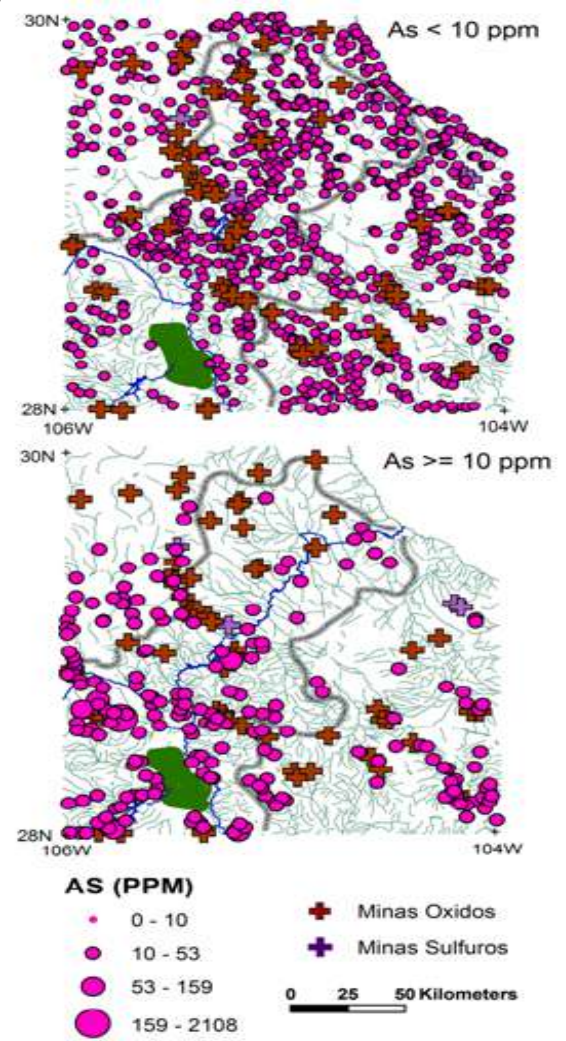

Figura 5. Concentraciones de fondo (mapa superior) y enriquecidas (mapa inferior) para Cu. Las minas están marcadas con cruces (rojas para óxidos, moradas para sulfuros). El mapa muestra además el río Conchos y su cuenca, y el sistema de riego 005 (verde).

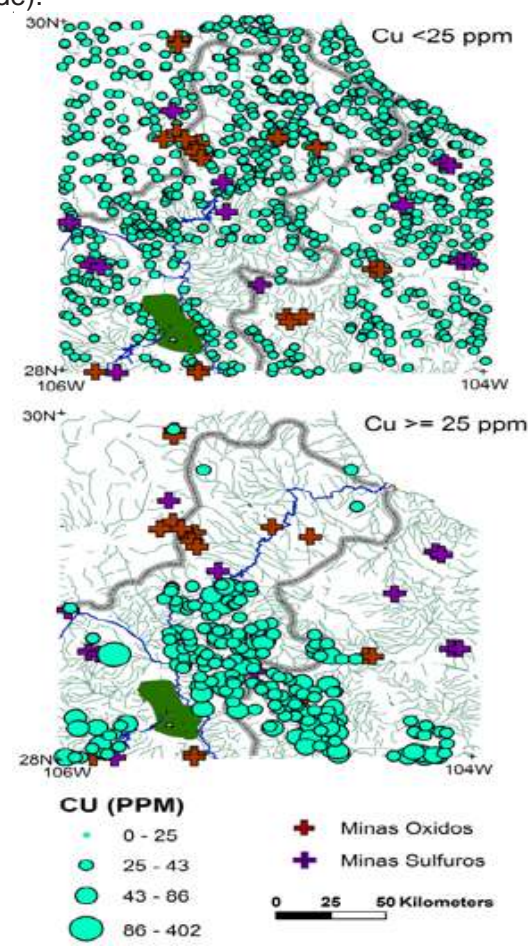

Figura 4. Concentraciones de fondo (mapa superior) y enriquecidas (mapa inferior) para Ba. Las minas están marcadas con cruces (rojas para óxidos, gris para sulfatos). El mapa muestra además el río Conchos y su cuenca, y el sistema de riego 005 (verde).

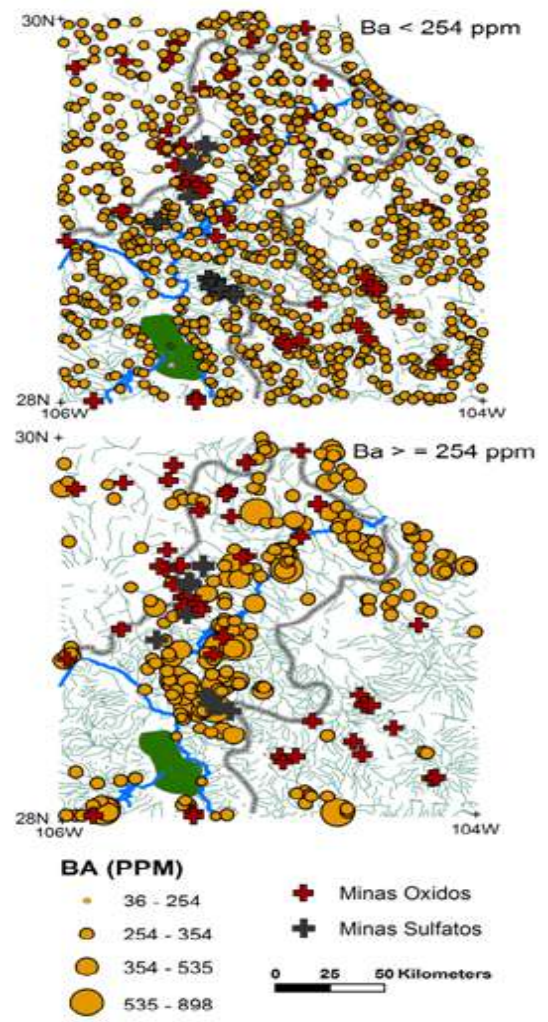

Figura 6. Distribución de concentraciones anómalas para As y $\mathrm{Ba}$ (círculos). Las minas mostradas incluyen depósitos minerales de metales (cruz roja), óxidos (cruz gris) y barita (cruz negra), mientras que las minas en operación están marcadas con un rombo.

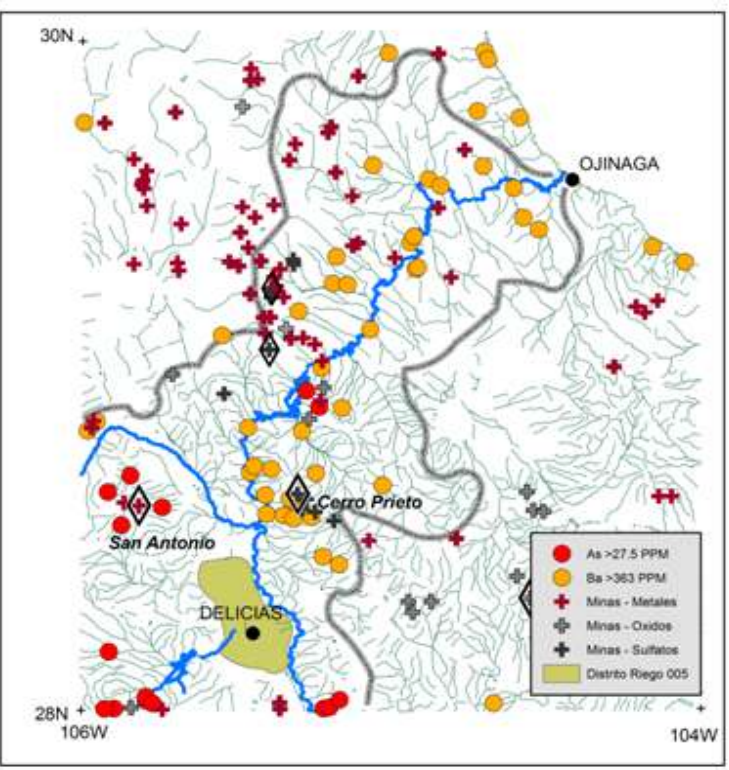


Mélida Gutiérrez, Enrique Carreon: Contenido de As, Ba, y Cu en sedimentos y su asociación con depósitos minerales en el noreste de Chihuahua

Cuadro 2. Numero de muestras de concentración de fondo natural presentes en la zona norte y zona sur del área de estudio.

\begin{tabular}{cccc}
\hline & $\begin{array}{c}\text { Zona Norte } \\
\text { No. puntos de } \\
\text { muestreo }\end{array}$ & $\begin{array}{c}\text { Zona Sur } \\
\text { No. puntos } \\
\text { de muestreo }\end{array}$ & $\begin{array}{c}\text { Diferencia } \\
\text { Significativa* }\end{array}$ \\
\hline As & 435 & 386 & No \\
Ba & 371 & 450 & $\mathrm{Si}$ \\
Cu & 476 & 347 & $\mathrm{Si}$ \\
\hline
\end{tabular}

* usando el criterio estadístico G-log verosimilitud; $G=2$ [ Efi In fi - Efi ln Fi ]

La asociación entre valores anómalos de As, Ba, Cu y depósitos minerales es evidente por simple inspección visual en la Figura. 6, donde las concentraciones anómalas de As y Ba están representadas por círculos. Las concentraciones anómalas de Cu se omitieron para simplicidad de la gráfica, además de que la mayoría de las concentraciones anómalas de Cu ocurren simultáneamente con las de As. En la Fig. 6 se puede observar que varios de los valores anómalos de As se encuentran directamente aguas abajo de zona minera San Antonio (mineral de Santa Eulalia), y varios valores anómalos de $\mathrm{Ba}$ aguas abajo de zonas mineras de barita (mina Cerro Prieto).

A diferencia de las concentraciones anómalas, el patrón de distribución obtenido para valores enriquecidos no-anómalos no se distribuyó necesariamente alrededor depósitos minerales, y cada uno de los elementos utilizados generó un patrón diferente. Algunas minas (probablemente las más pequeñas) no generaron valores enriquecidos 0 anómalos en sus alrededores, tal vez como resultado de tener menos cantidad de jales o una concentración menor del elemento en cuestión.

El arsénico mostró una asociación estrecha con la presencia de minas de sulfuros, mientras que en el caso de $\mathrm{Ba}$ y $\mathrm{Cu}$ las concentraciones parecen ser afectadas, además de la presencia de minas, por otros procesos tales como la erosión de minerales conteniendo Ba y Cu que hace que contenido de estos elementos en el sedimento se encuentren esparcidos sobre una zona extensa y en concentraciones suficientemente bajas como para no ser clasificados como depósito mineral. La abundancia de Ba en la parte norte y áreas cercanas a los ríos se puede deber, en parte, a su mayor facilidad para ser transportado por agua de escorrentías. En el caso del cobre, la mayoría de los valores naturales enriquecidos ${ }^{3}$ percentil 80) están localizados en la parte sur del área de estudio, sin aparentemente relación con la presencia de minas, sugiriendo que otros procesos como geología de los afloramientos y topografía contribuyen de manera importante a su contenido y distribución.

En general, el análisis de distribución 
arrojó como conclusión que las concentraciones de fondo natural coincidieron con áreas apartadas de ciudades, actividades agrícolas y mineras, mientras que los valores anómalos se localizaron en lugares cercanos y aguas abajo de depósitos minerales, como las minas de San Antonio (As y $\mathrm{Cu}$ ) y Cerro Prieto $(\mathrm{Ba})$. El patrón de concentraciones naturales enriquecidas varió para cada elemento y además de ofrecer un valor base con el cual comparar concentraciones naturales enriquecidas para esta área mostró lugares donde este elemento se acumula en forma natural.

\section{Conclusiones}

El abundante número de muestras disponibles hizo posible identificar con claridad los dos componentes de distribuciones normales y por consiguiente separar con certeza las concentraciones naturales de fondo de las concentraciones enriquecidas.

Para los tres elementos estudiados, los valores anómalos se encontraron en la cercanía a minas en operación. Los valores naturales enriquecidos presentaron un patrón más complejo, y a diferencia de As y $\mathrm{Ba}$, la relación entre $\mathrm{Cu}$ y depósitos minerales fue menos evidente, mostrando las concentraciones más altas en la zona sur del área, sin encontrársele explicación a este patrón de concentraciones.

Concentraciones de fondo para áreas naturales no contaminadas y concentraciones naturales enriquecidas son parámetros base sumamente útiles en la predicción de los efectos de actividades futuras puedan ejercer en suelos, sedimentos, y consiguientemente en aguas naturales.

\section{Literatura citada}

Agency for Toxic Substances and Disease Registry (ATSDR): 2007 ToxFAQs ${ }^{\mathrm{TM}}$ for Barium. http://www.atsdr.cdc.gov/ tfacts24.html

ArZABALA-Molina J., 2005. Correlación estratigráfica de la Cuenca de Chihuahua y su relación con los yacimientos minerales. Boletín Técnico Mayo-Junio 2005. Servicio Geológico Mexicano. México.

BRECKENRIDGE R.P. and A.B. Crockett. 1995. Determination of background concentrations of inorganics in soils and sediments at hazardous waste sites. EPA Office of Solid Waste and Emergency Response, EPA/540/5-96/500. http:// www.epa.gov/tio/tsp/download/bckgrnd.pdf

CLARK K.F. AND F.E. DE LA FuENTE, 1978. Distribution of mineralization in time and space in Chihuahua, Mexico. Mineralium Deposita (Berl.) 132, 27-49.

DIARIO OFICIAL DE LA FEDERACIÓN (DOF). 1996. NOM-147-SEMARNAT/ SSA1-2004. México: Modificada 22 nov. 2000 www.salud.gob.mx/unidades/cdi/nomssa.html

DiARIO OfICIAL DE LA FEDERACIÓN (DOF), 2007. NOM-147-SEMARNAT/ SSA1-2004. México: Publicado el 2007-03-02. Www.economia-noms.gob.mx/

Environmental Protection Agency (EPA), 2001. Streamlined WaterEffect Ratio Procedure for Discharges of Copper. EPA Office of Water, EPA-822-R-01-005, www.epa.gov/waterscience/ criteria/copper/copper.pdf

Förstner U.,and G.T.W. Wittmann. 1981. Metal Pollution in the Aquatic Environment. Springer -Verlag Berlin, New York, 486pp.

Flemming, C.A, and J.T. Trevors. 1989. Copper toxicity and chemistry in the environment: A review. Water, Air and Soil Pollution, 44, 143-158

Gutiérrez, M., M.T. Alarcon-Herrera, and L. Camacho. Geographical distribution of arsenic in sediments within the Rio Conchos basin, Mexico. Environmental Geology, en prensa.

Gutiérrez, M., C. Martinez-Pina, J. Luo, and K. Mickus, 2008. Geochemical processes contributing to the contamination of soil and surface waters in the Rio Conchos basin, Mexico. Geosphere, 4:600-611.

GutiérRez-Ruız, M., F.M. Romero y G. González-Hernández. 2007. Suelos y sedimentos afectados por la dispersión de jales inactivos de sulfuros metálicos en la zona minera de Santa Barbara, Chihuahua, México. Revista Mexicana de Ciencias Geológicas, 24:170-184.

Hernandez-Garcia Y, Sosa-Cerecedo M, Quintana-Martinez C, SotoCRuz R, 2007. Evaluacion de la contaminación por metales pesados y arsénico en agua en embalses del estado de Chihuahua, Mexico. Memorias en Extenso. VI Congreso Internacional y XII Nacional de Ciencias Ambientales. Chihuahua, Chih. Mexico, 217-221.

Instituto Nacional de Estadística, Geografía e Informática (INEGI), 1999. Estudio Hidrológico del Estado de Chihuahua. INEGI, Aguascalientes, México, 222 pp

LoNG E.R., and L.G. Morgan, 1990. The potential for biological effects of sediment-sorbed contaminants tested in the National status and trends program. NOAA Technical Memorandum NOS OMA 52, 346 pp.

Mosier, D.L. 1986. Descriptive model of epithermal Mn, In Cox D.P. and D.A. Singer (Eds), Mineral deposit models: U.S. Geological Survey Bulletin 1693:165

MülleR, G. 1979. Schwermetalle in den sedimenten des Rheins - Veränderungen seit 1971. Umshau 79, 778-783.

PARADIS, S., G.J.Simandl, D.MacIntyre, and G.J.Orris, 1998, 
Sedimentary-hosted, stratiform barite, in Geological Fieldwork 1997: British Columbia Geological Survey Paper 1998-1, p. 24f.1-24f.2.

Puga ,S., M. Sosa, T.Lebgue, C. Quintana and A. Campos, 2006. Heavy metals pollution in soils damaged by mining industry. Ecologia Aplicada, 5:149-155.

PuRdeY, M. 2004, Chronic barium intoxication disrupts sulphated proteoglycan synthesis: a hypothesis for the origins of multiple sclerosis. Medical Hypotheses. 62:746-54.

SAN JUAN, C. 1994. Natural Background soil metals concentrations in Washington State. Publication No. 94-115, Toxics Cleanup Program. Olympia, WA 98504.

Secretaria de Medio Ambiente y Recursos Naturales (SEMARNAT) , 2007. NOM-147-SEMARNAT/SSA1-2004. Publicada 200703-02. http://normas.economia.gob.mx/normas/normas.do
Servicio Geológico Mexicano (SGM). 2001. Paquete Digital Interactivo Ciudad Delicias H3-11 y Ojinaga H13-8. Escala 1:250,000. Pachuca, Hgo. México. http:// www.coremisgm.gob.mx/inicio.html

Tessier, A., P. Campbell, and M. Bisson, 1979. Sequential extraction procedure for the speciation of particulate trace metals. Analyt Chem. 51:844-851.

VoIGt, H.J., S. Hannappel, R. Kunkel, and F. Waendland, 2005. Assessment of natural groundwater concentrations of hydrogeological structures in Germany. Geologija, 50:35-47.

Wones, R. G., B. L. Stadler, and L. A. Frohman, 1990. Lack of effect of drinking water barium on cardiovascular risk factor. Environ. Health Perspect. 85:1-13 O)

Este artículo es citado así:

GutiérRez MélidA, Enrique Carreon. 2008. Contenido de As, Ba, y Cu en sedimentos y su asociación con depósitos minerales en el noreste de Chihuahua. TECNOCIENCIA Chihuahua 2(2): 40-55.

\section{Resúmenes curriculares de autor y coautores}

MéLIDa GutiérRez. Es originaria de Delicias, Chihuahua. Cursó su maestría en la Universidad de Karlsruhe, Alemania, obteniendo su grado en sistemas biológicos de tratamiento de agua en 1979. Estudió el doctorado en geohidrología en la Universidad de Texas en El Paso, de donde se graduó en 1992. Ha impartido clases en el Instituto Tecnológico de Monterrey-Campus Guaymas, la Universidad del Estado de Nuevo Mexico - Las Cruces, y durante los últimos quince años, en la Universidad del Estado de Missouri, en Springfield, Missouri. Actualmente imparte los cursos de geología física, ciencias de la tierra, y geoquímica. Su investigación se ha enfocado a la calidad del agua y geoquímica de interacciones entre roca y agua, especialmente los sistemas cársticos en el sur de Missouri y el río Conchos en Chihuahua. Su investigación en el rio Conchos incluye, además de la química del agua y de sedimentos, la educación ambiental. Con este motivo viaja a Chihuahua con frecuencia y mantiene nexos de colaboración con colegas de instituciones educativas en el estado.

Enrique Carreon Hernandez. Es Médico Veterinario Zootecnista por la Universidad Autónoma de Zacatecas, Maestro en Ciencias en manejo y conservación de vida silvestre por el Colegio de Postgraduados y candidato a Doctor en Filosofía por la Universidad Autónoma de Chihuahua en manejo y conservación de recursos naturales. Actualmente labora en Protección de la Fauna Mexicana, A.C. siendo su área de trabajo el manejo y conservación de recursos naturales, percepción remota y sistemas de información geográfica. 